\title{
Centaur: a mobile dexterous humanoid for surface operations
}

\author{
Fredrik Rehnmark ${ }^{\mathrm{a}}$, Robert O. Ambrose ${ }^{\mathrm{b}}$, S. Michael Goza ${ }^{\mathrm{b}}$, Lucien Junkin ${ }^{\mathrm{b}}$, Peter D. Neuhaus ${ }^{\mathrm{c}}$, \\ Jerry E. Pratt ${ }^{c}$ \\ ${ }^{a}$ Lockheed Martin Space Operations, Houston, TX 77058 \\ 'NASA Johnson Space Center, Houston, TX 77058 \\ ${ }^{\mathrm{c}}$ Institute for Human and Machine Cognition, Pensacola, FL 32502
}

\begin{abstract}
Future human and robotic planetary expeditions could benefit greatly from expanded Extra-Vehicular Activity (EVA) capabilities supporting a broad range of multiple, concurrent surface operations. Risky, expensive and complex, conventional EVAs are restricted in both duration and scope by consumables and available manpower, creating a resource management problem. A mobile, highly dexterous Extra-Vehicular Robotic (EVR) system called Centaur is proposed to cost-effectively augment human astronauts on surface excursions. The Centaur design combines a highly capable wheeled mobility platform with an anthropomorphic upper body mounted on a three degree-of-freedom waist. Able to use many ordinary handheld tools, the robot could conserve EVA hours by relieving humans of many routine inspection and maintenance chores and assisting them in more complex tasks, such as repairing other robots. As an astronaut surrogate, Centaur could take risks unacceptable to humans, respond more quickly to EVA emergencies and work much longer shifts. Though originally conceived as a system for planetary surface exploration, the Centaur concept could easily be adapted for terrestrial military applications such as de-mining, surveillance and other hazardous duties.
\end{abstract}

Keywords: robot, Centaur, EVA, Robonaut, NASA, telemanipulation, telerobotics

\section{INTRODUCTION}

In order for NASA to pursue its aggressive agenda of returning humans to the Moon for long duration stays in preparation for an eventual mission to Mars, EVA systems must evolve beyond current configurations. Spacewalks and surface excursions are critical for assembly, inspection and maintenance operations but they demand much preparation, consume significant crew time and pose a resource bottleneck to everything that depends on them. Risky, expensive and complex, EVAs are restricted in both duration and scope by consumables and available manpower. Moreover, EVA tools and equipment designed for use aboard the International Space Station operate in a relatively well-structured 0 -g environment that enables crewmembers to maneuver massive pieces of hardware with ease. In comparison, gravitational forces and rugged terrain will significantly degrade EVA performance and mobility on the lunar surface.

Unmanned vehicles have been deployed in hazardous environments ranging from the ocean floor to the surface of Mars. Long recognized as invaluable tools for scientific discovery, they are now beginning to prove themselves on the modern battlefield and in terrorist threat response operations. As these machines become more capable and affordable, their roles will expand to assume even more risks unacceptable to humans and perform extended missions well beyond human endurance.

Recent advances in miniaturization and embedded processing have revolutionized human-machine interfaces and advanced the state-of-the-art in robotic telemanipulation. It is now possible to project humanlike dexterous manipulation and tool-use capabilities into remote, hostile environments by means of teleoperated robotic surrogates like NASA's Robonaut system ${ }^{1}$. Such systems allow their human operators to perceive remote locations and perform complex mission objectives while safely situated in a shirtsleeve environment. Through their use, Intra-Vehicular Activity (IVA) humans and ground controllers could participate in EVAs in new and meaningful ways. Multi-agent EVA teams combining the information-gathering and problem-solving skills of human astronauts with the survivability and physical capabilities of diverse robot archetypes were featured in President George W. Bush's national vision for US space exploration, announced on January 14, 2004 (http://www.nasa.gov). 


\section{SYSTEM CONCEPT}

Robotic planetary explorers come in many shapes and sizes ${ }^{2,3,4}$, are highly adapted to their target operating environments and, with a few notable exceptions, are generally not designed to interact with humans. The recent successes of the NASA MER (Mars Exploration Rover) missions have highlighted what can be achieved with a single, subdexterous manipulator arm mounted on a semiautonomous vehicle with a passive suspension. Because it must move slowly and tread lightly to conserve power, however, this class of robotic explorer would not be well-suited to accompanying a human astronaut in the field.

A new class of robot taxonomy for planetary surface exploration is proposed, pairing advances in upper body dexterity with unique mobility features in a configuration dubbed the Centaur. As its name implies, the robotic Centaur has the upper body of a humanoid integrated with a four-legged mobility platform that can propel the system over rough terrain, while carrying payloads such as scientific equipment and geological samples or potentially even transporting astronauts. Articulation in the lower body will allow the robot to "crouch" close to the ground and control body attitude in rough terrain. With backup life support systems on board, Centaur can come to the aid of an astronaut in trouble until other humans arrive. The robot's physical endurance in maintaining stable grasps and bending over to inspect and retrieve objects on the ground will greatly exceed that of a suited astronaut. Able to use many handheld tools designed for humans, Centaur could serve as a highly flexible member of a multi-agent assembly team with the unique capacity to collaborate naturally with humans. Though originally conceived as a system for planetary surface exploration, the Centaur concept could easily be adapted for terrestrial military applications such as de-mining, surveillance and other hazardous work.

\subsection{Anatomy}

As shown in Fig. 1, the Centaur configuration features a hybrid leg/wheel system allowing it to handle irregular terrain while the articulated 3-DOF (Degree-Of-Freedom) waist coordinates torso posture to maintain overall vehicle stability by managing the system's center of gravity (CG). By "leaning" appropriately, the upper body can position the system CG over any of the triangles formed by any of the four combinations of three legs, allowing the Centaur to lift its fourth leg over obstacles on the ground. The robot can then drive forward onto ledges, curbs, or shelves that would defy simpler wheeled systems which are normally limited to rolling over obstacles less than half a wheel diameter in height. A key performance metric for locomotion will be to operate over terrain obstacles that are taller than 1 wheel diameter. The leg/wheel hybrid can also serve as an active suspension system, leveling the system over cross slopes, leaning into steep hill climbs and providing a smooth ride for onboard avionics, payloads and passengers.

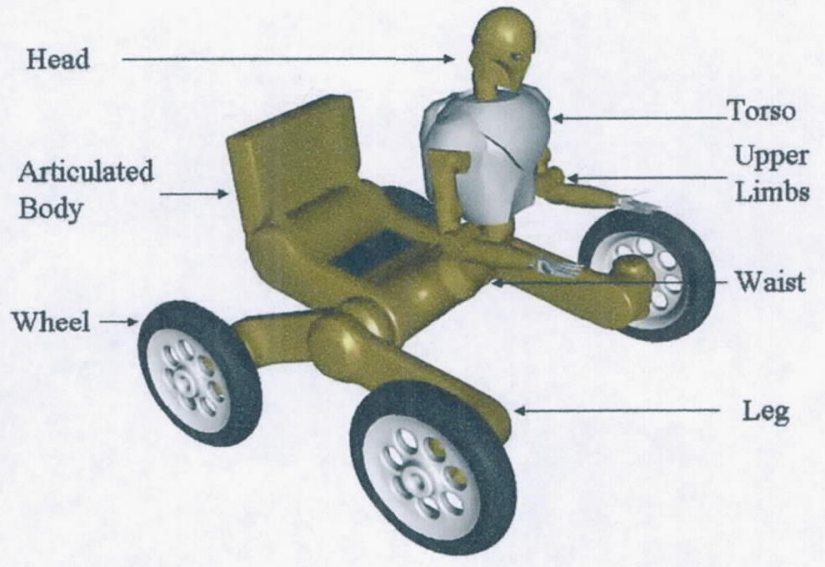

Figure 1. Gross anatomy of the Centaur system.

A derivative of NASA's Robonaut system, the Centaur upper body is humanoid in form. The Robonaut system is one of the most advanced dexterous robots in the world and has been demonstrated handling precision instruments, working 
with flexible materials, opening luggage and operating pistol grip power tools. Designed to work on the International Space Station, this machine has set new standards for dexterous robots, and will be an ideal complement to the agility of the Centaur's lower body. Self-propelled and tetherless, the Centaur will be able to project a remote human's dexterity and problem solving capabilities into dangerous environments, either alone or in support of human teammates.

Although controlled in a coordinated fashion, Centaur's upper and lower bodies are distinct, self-contained robots in their own right. The torso may draw power from the vehicle's batteries but is also equipped with its own.

Correspondingly, the lower body may take advantage of the unobstructed view from the humanoid's head but also carries its own imaging devices for navigation and obstacle detection. To preserve extensibility and modularity, the interface between the torso and the vehicle is simple and uncluttered, facilitating separation and reconfiguration. Depending on the service environment and tasks to be performed, the torso can be mated with different mobility platforms while the vehicle may carry other utilities into the field.

\subsection{Telemanipulation}

Central to Centaur's design is the anthropomorphic Telemanipulation Work System (TWS), based on Robonaut technology developed by NASA and Lockheed Martin Space Operations. In its simplest form, the TWS comprises three electromechanically actuated subsystems: a multipurpose robotic end-effector (hand), a dexterous robotic manipulator (arm) and a stereo camera pair mounted on an articulating platform for live video feedback to the operator (head and neck).

Coupled with a wearable teleoperator interface known as FITT (Full-Immersion Telepresence Terminal), the anthropomorphic form of the TWS allows an intuitive, one-to-one mapping between human (master) and robot (slave) motions (see Fig. 2). Extremely easy to use, FITT offers even novice operators a virtually transparent interface for efficiently controlling the TWS. The more seamless this fusion of human cognitive abilities (including eye-hand coordination, perception, etc.) and machine physical abilities becomes, the more productive the human-robot system can be. Where appropriate, the TWS may also be operated using more conventional interfaces.

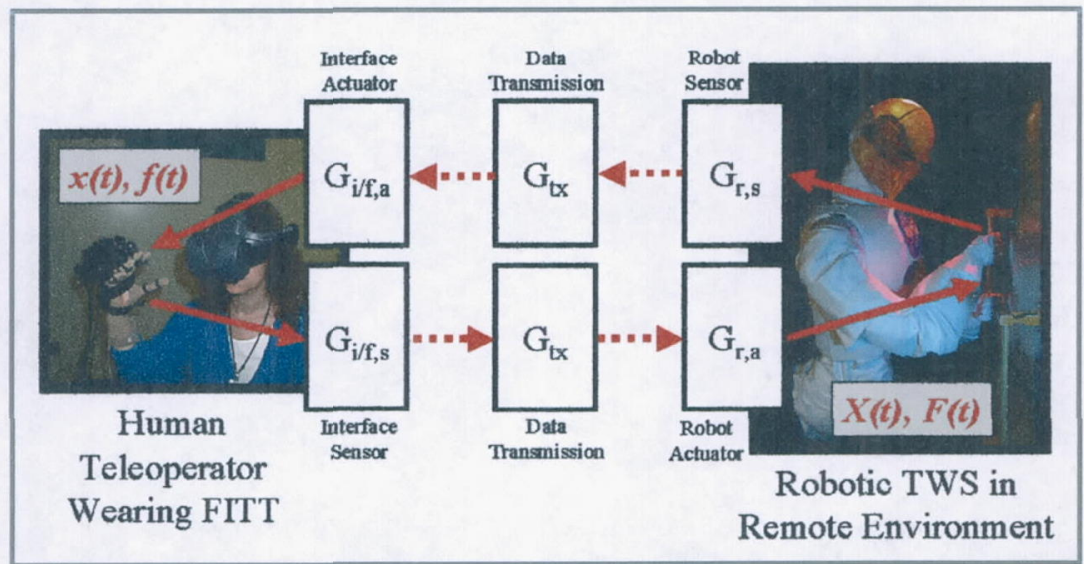

Figure 2. The Full-Immersion Telepresence Terminal is used to control the anthropomorphic Telemanipulation Work System.

For maximum flexibility, Centaur, like Robonaut, features a dual-arm TWS with a high strength-to-weight ratio and sophisticated humanoid hands capable of using ordinary handheld tools and manipulating flexible materials (e.g., fabric, hoses, wire, etc.) and common interfaces such as doorknobs which would frustrate conventional robotic grippers. The TWS is mounted on a three degree-of-freedom waist in a bifurcating arrangement that greatly expands its reachable workspace, enabling access to storage compartments, tool caddies and other payloads carried on the vehicle chassis. Centaur's familiar humanoid form facilitates collaboration with adjacent humans. Semiautonomous control modes allow the system to visually track and follow humans while responding to spoken commands and gestures. This may be in addition to some related task objective, like cooperating with a human coworker to maneuver a long, unwieldy object. 


\subsection{Locomotion}

While FITT is useful for teleoperation of the Centaur's upper body, an operator controlling the robot's lower body will require a different interface. There are six motions, or degrees of freedom, of the base that need to be controlled on a continuous basis. Body attitude (pitch, yaw, roll) relative to terrain is determined by robot leg posture and can be commanded with a rate-based hand controller (joystick) instrumented for angular deflections. Body translations can be commanded with a second rate-based hand controller instrumented for angular deflections in two directions, corresponding to planar translations (enabled by all-wheel steering), and linear deflection in a third direction, adjusting body height.

Widely employed by NASA in controlling precision motions of manned space vehicles and robotic arms, the dual hand controller configuration obviously precludes simultaneous teleoperation of the upper and lower bodies by a single operator. Another option is to control steering and acceleration with foot pedals, a technique employed on NASA's mobile Robonaut ${ }^{5}$ (see Fig. 3), while allowing the vehicle to manage body attitude and height automatically in an effort to distribute ground pressure evenly between the four wheels. This configuration allows a single operator, with some practice, to coordinate upper and lower body motions.

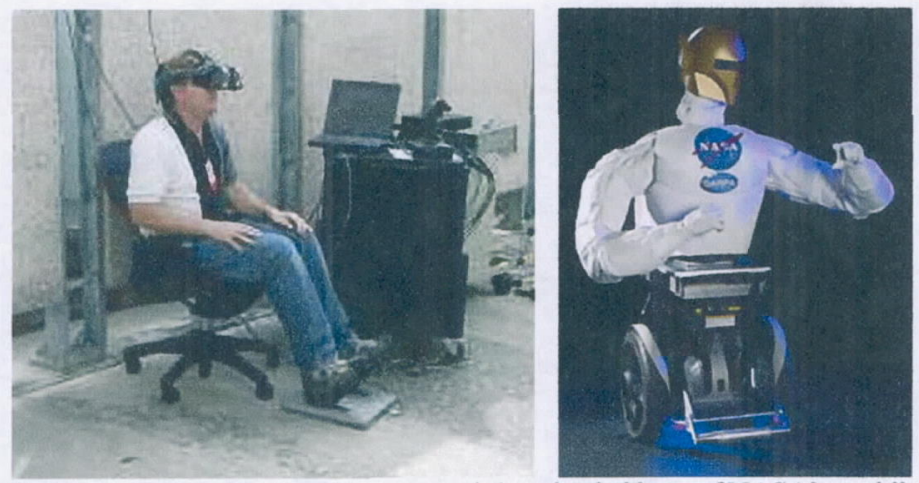

Figure 3. A teleoperator uses foot pedals to control the wheeled base of NASA's mobile Robonaut.

Coordination between Centaur's upper and lower bodies is an integral part of the system's concept of operations. Much like a human riding an ATV, Centaur's upper body compensates for motion of the chassis as the lower body conforms to terrain, improving the vehicle's overall stability margins and its ability to track targets while in motion. During high speed traverses that may upset the stability of the robot even if the system center of gravity is maintained above the vehicle's ground support polygon, dynamic compensation will be employed in addition to center of gravity management. Given a command from the user interface, a simplified dynamic model of the robot will be simulated in real-time to predict the expected change in the center of pressure on the ground. If the computed center of pressure lies too close to the edges of the support polygon, the command will be modified before being sent to the robot. For example, if the teleoperator commands a fast turn, the upper body will lean into the turn, preventing the robot from tipping. When the risk of rollover is particularly high, as in high speed traverses, the upper body can be retracted into the vehicle chassis.

\section{CAPABILITIES FOR PLANETARY SURFACE EXPLORATION}

\subsection{Upper Body}

NASA has developed one of the most sophisticated dexterous robots ever built, called Robonaut. First appearing in 1998, this system is currently under development at NASA's Johnson Space Center with the original goal of aiding astronaut crews during Extra-Vehicular Activity (EVA) missions. This role motivated the requirement that the robot be able to handle ordinary tools designed for humans (see Fig. 4). Collaborating with NASA, DARPA/IPTO's MARS Program has further invested in the system to automate its operation and allow its control in a supervisory form of remote operation that requires a low level of human intervention. Its ability to autonomously reach and grasp irregular objects using stereo vision, haptic sensing, and force feedback control has now matured, opening the door for application on planetary surfaces across time delays and distances that defy the teleoperation modes available for orbital 
EVA, and where complex tasks as a member of a multi-agent team including humans requires a high degree of independence.
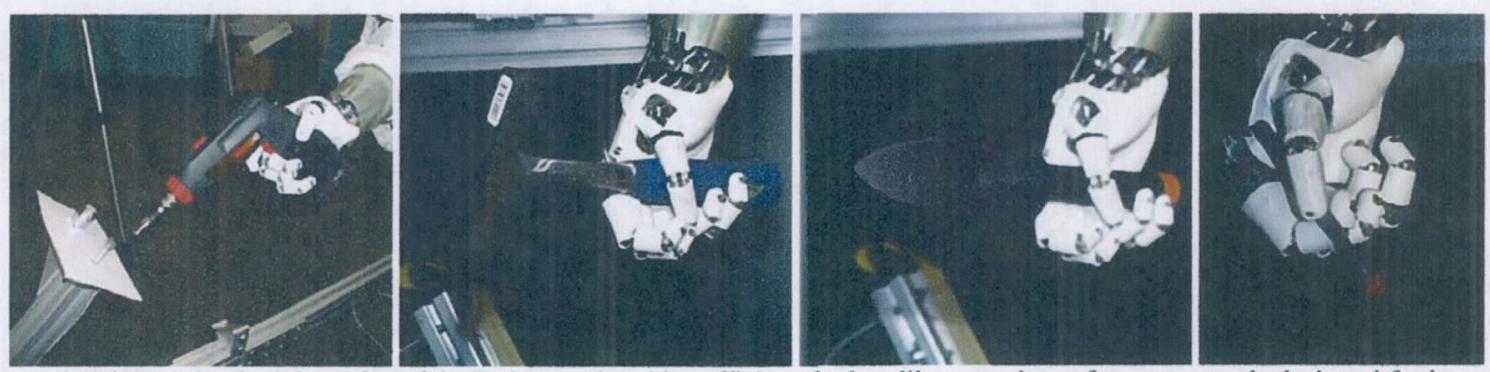

Figure 4. The mechanical dexterity of the Robonaut hand is sufficient for handling a variety of common tools designed for humans including (from left to right) power drills, picks, shovels, and tweezers $(0.5 \mathrm{~cm}$ rock).

The anthropomorphic form of the Robonaut upper body should not be discarded as purely cosmetic. Biologically inspired in its design, Robonaut shares only partial human form, with efficient dual arm dexterity as the real driver in its design. The integrated design of Centaur's science work bench and wheeled rover have no anthropomorphic heritage, but have also been driven by productivity goals, with form following function ${ }^{6}$. The placement of the arms on a 3-DOF waist allows the dexterous workspace of this upper body to be positioned for best effect, either bending to reach for the ground or rotating fully rearward to reach the robot's work deck. Waist mobility allows the relatively short arms to reach more widely separated targets in the rover's immediate vicinity than if the arms were fixed directly to the rover chassis.

Figure 5 shows the Robonaut upper body, mounted on a fixed pedestal, performing a planetary geology experiment in 2001. This experiment was conducted with the robot and a human in a spacesuit performing the tasks separately to compare their capabilities and identify teaming opportunities ${ }^{7}$. Robonaut was able to complete all the attempted tasks, though at a slower pace than the person. The range of human vs. robot task completion times varied from 1:2 up to $1: 20$, depending on the complexity of the tasks.
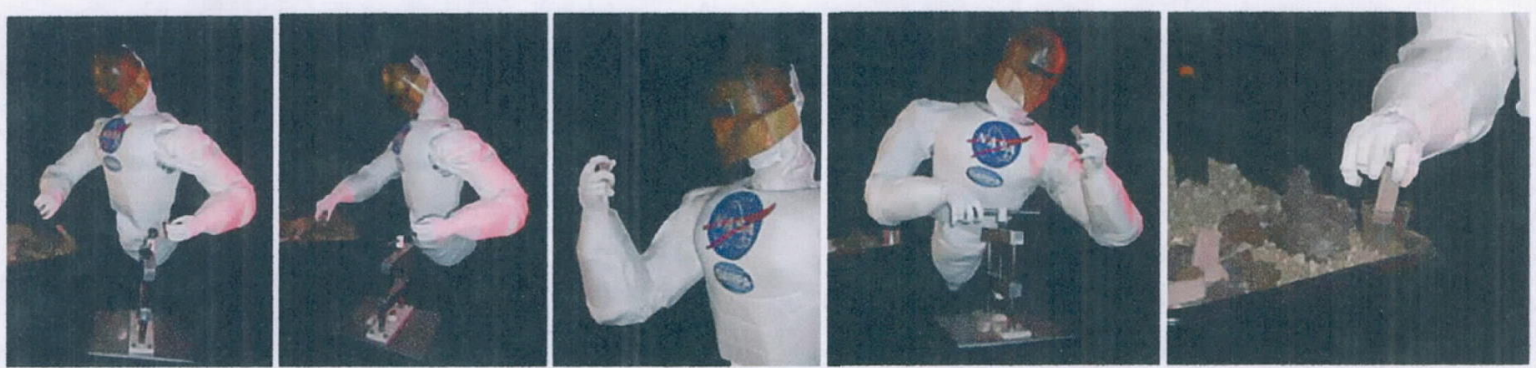

Figure 5. Robonaut performing steps in a geological sample collection experiment. Steps shown include (from left to right) digging, acquisition, inspection, cleaving and collection.

The geology experiment tasks were a mix of sample acquisition, sample handling, sample preparation, and sample analysis functions, allowing the team to link relative performance to very specific functional "primitives". Each step was part of a larger task, which sought to retrieve a $15 \mathrm{~cm}$ rock sample out of packed gravel $(0.1$ to $0.5 \mathrm{~cm}$ aggregate) that had a Mars analog density and texture. After exposing a suitable sample by digging with a small hand shovel, the sample was grasped by hand, and placed before cameras for imaging. After imaging, the rock was either discarded or passed to the next step, where the rock was split using a rock-cracking tool. This was a complex, two-handed task, requiring the sample be placed in the jaws of the cracker, and then operating a crank with the other hand. After a piece of the rock was cleaved, the cut piece was grasped and repositioned in front of a sensor for imaging, after which it was either discarded or placed in a storage container. This complete sequence of tasks was completed by Robonaut in about 10-14 minutes, with the suited human completing the tasks in 1-3 minutes. 
This general dexterous manipulation ability to work with rocks, hand tools, bench tools, and sample containers fully utilizes the Robonaut dual arm, high DOF system. The system's anatomy includes two 7 DOF arms of human scale, 2 hands each with 12 DOF and five fingers, a set of four cameras mounted on 2 DOF eye verge mechanisms protected within the helmet, which has a 2 DOF neck, and the whole torso is mounted on a 3 DOF articulated waist.

\subsection{Lower Body}

More than just a rigid chassis with wheels attached, the Centaur's lower body is an articulated series of segments that allow it to conform to challenging terrain and maintain an upright attitude in the face of cross slopes and steep climbs. This articulated approach allows greater flexibility in managing the system center of gravity (CG) by expanding the suspension's travel (stroke or workspace) to allow the wheels to maintain traction and a stable stance. Each of its four wheels are independently steered and powered, enabling turn-in-place maneuvers as well as multidirectional translation. Each wheel is mounted on a single-DOF articulating arm, enabling active suspension for a smooth ride for onboard subsystems and cargo as well as body attitude control in rough terrain. As a mobility system, performance is comparable whether the vehicle is traveling in forward or reverse. The proposed system would have a multi-segment lower body optimized to meet the workspace needs of the upper body, enabling diverse capabilities such as sample acquisition, processing and analysis. Self-inspection, servicing, and stowage issues must also be considered.

The proposed configuration, shown conceptually in various poses in Figures 6 through 8, has an articulated design with a work deck and upper body mounted on a common lateral axle. All four wheels are mounted on "legs" that are also mounted to this common hub, allowing a wide range of motion options. The design has been developed using modern CAD tools and animation products, and used in a workspace study to identify segment proportions that fulfill system goals of high and low reach envelopes for sample acquisition, and allowing the upper body to rotate fully around to interact with science instruments located behind it on the work deck. The following discussion highlights the robot in specific poses that demonstrate many of these new capabilities.

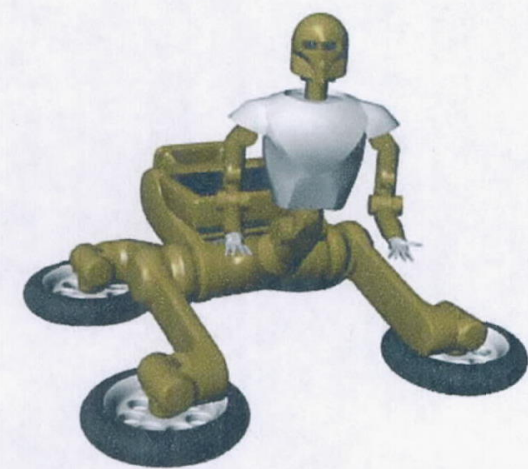

Figure 6a. Centaur stabilized with low ground pressure suitable for working in loose regolith.

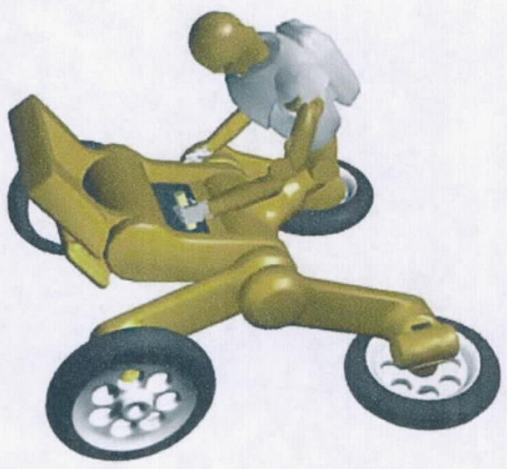

Figure $6 \mathrm{~b}$. Centaur accessing its own onboard cargo area.

Figure 6a shows the Centaur in a stationary pose, with wheels turned down to stabilize the body for sustained site work. The wheel/ankle design allows it to accommodate uneven terrain, and still achieve this stable stance, enabling delicate work with powders, fluids or other samples that require a steady hand for manipulation. This would also be the ideal stance for stabilizing a core drill mounted on the robot's rear implement plate. Figure $6 \mathrm{~b}$ shows the Centaur processing samples using various instruments on its workbench. The body can rotate fully around to face rearward and bend at the waist, allowing it to reach all locations on the body with either or both arms. In this case, two of the wheels were turned flat to stabilize the base. The work deck opens and closes, allowing it to be sealed for protecting instruments, tools and samples. 


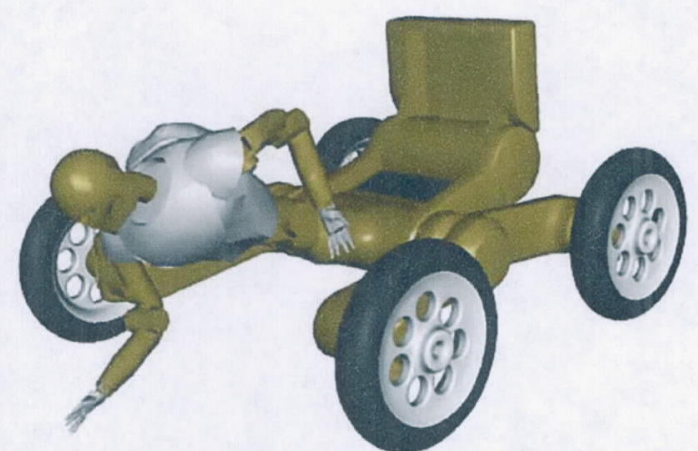

Figure 7a. Centaur bending at the waist to reach objects on the ground.

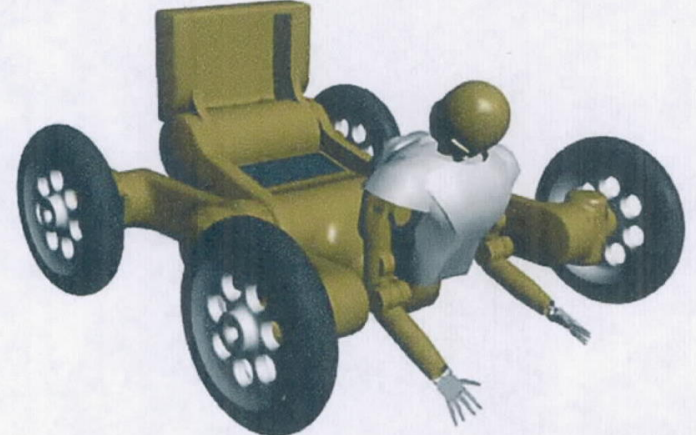

Figure $7 \mathrm{~b}$. Centaur crounching down to interact with objects on the ground.

Figure 7a shows the system with a nominal reach, acquiring a sample. Notice that the wide stance of the forward wheels provides a "well" within which the upper body can move freely without constraint. The reach is a coordinated motion of the arm and waist, mounted on the lower body that has been posed with the legs in a horizontal stance that temporarily trades ground clearance. Figure $7 \mathrm{~b}$ shows the Centaur reaching low samples by using its front legs to lower its upper body closer to the ground. This crouching position allows Centaur to retrieve large samples using two hands without having to bend at the waist. Like a human, the Centaur will be able to pickup large loads without "back strain", by lifting with its powerful leg motors rather than its waist. This position also allows the system to have a large and overlapping workspace on the ground where the two arms can dig and work.

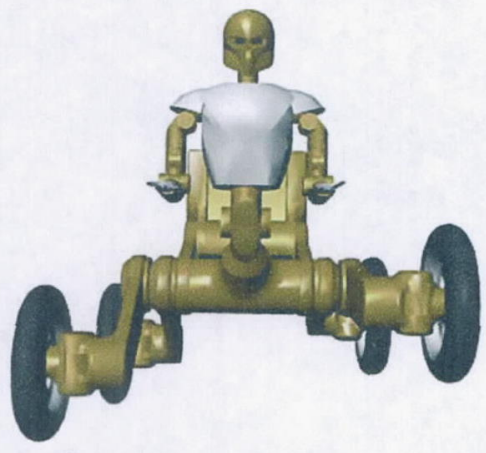

Figure 8a. Centaur traversing across a slope.

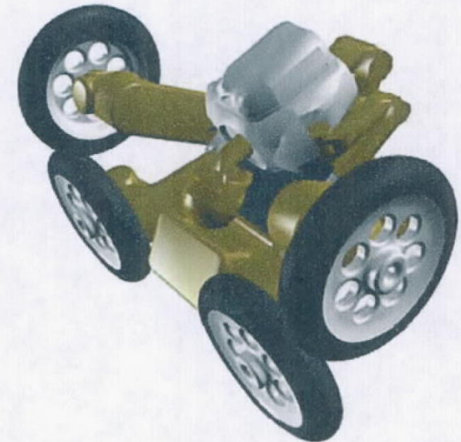

Figure $8 \mathrm{~b}$. Centaur in a stowed position.

Figure 8a shows the Centaur, face on, traversing a cross slope. The legs are independently actuated, allowing the vehicle ground clearance to be fully controlled to accommodate cross slopes, and level the upper body on inclines of up to 30 degrees. Leaning the upper body and manipulators will allow it to handle slopes of up to 45 degrees. Figure $8 \mathrm{~b}$ shows the CENTAUR in a stowed pose. This geometry allows the upper body to connect to the work deck for vibration support with the legs fully folded to minimize volume. The wheels have been indexed to further reduce volume, and provide flat exterior volumetric surfaces for mating with landers, pallets or aircraft stowage interfaces.

\section{MILITARY APPLICATIONS}

Numerous military programs have recognized the potential for robotic vehicles to support ground combat forces in the field, carrying supplies, weapons systems and mission-specific payloads into hostile environments. The UGCV (Unmanned Ground Combat Vehicle) prototype, developed for DARPA by Lockheed Martin Missiles and Fire Control (LMMFC), has demonstrated extreme maneuverability in rough terrain as well as unique climbing abilities (see Fig. 9). A robotic vehicle powered by hybrid-electric drive could maintain very low thermal and acoustic profiles and could 
station-keep for several weeks at a time without refueling. This could prove extremely useful in covertly monitoring enemy activity on the ground and in conducting stealthy nighttime operations behind enemy lines.
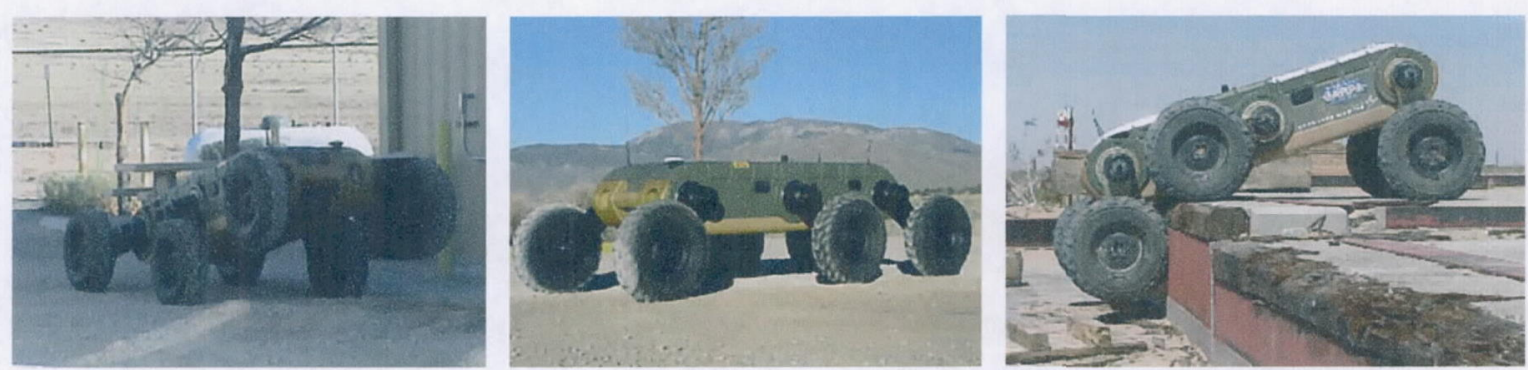

Figure 9. The UGCV is a highly capable robotic vehicle prototype built by LMMFC under contract to DARPA.

A new configuration called CENTAUR (Covert ENvoy for Telemanipulation And Unmanned Reconnaissance) is proposed to extend the possible uses of the Unmanned Ground Vehicle (UGV) as a human surrogate and assistant to the warfighter. The CENTAUR payload, shown in Fig. 10, incorporates general-purpose telerobotic manipulation and teleperception capabilities, allowing human operators not only to remotely observe dangerous environments, but also to take meaningful action. CENTAUR can operate in hostile and challenging environments with numerous hazards present including chemical and/or biological agents, high levels of background radiation and/or enemy fire.

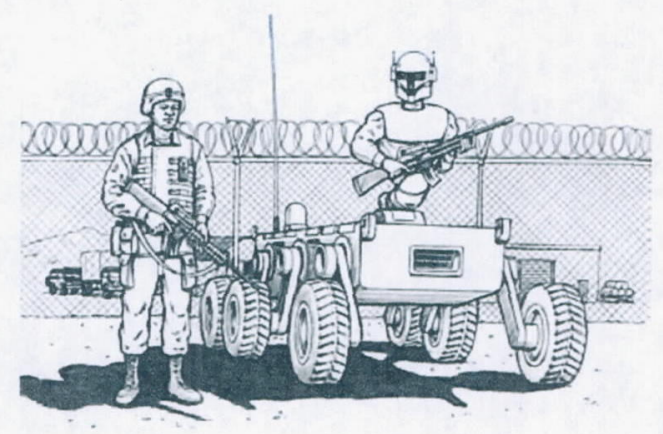

Figure 10. CENTAUR on sentry duty.

The proposed CENTAUR payload enables the following Unmanned Ground Vehicle roles:

1. loading/unloading gear at unmanned depots or forward positions

2. defusing/disposing of unexploded ordnance (landmines, IEDs, etc.)

3. precision infrastructure disruption via covert operations

4. medical evacuation ( $w /$ triage) from hostile territory

5. suspicious vehicle and package inspection at security checkpoints

6. refuel, repair or recover other unmanned vehicles

7. low profile scouting ahead of infantry

8. surveillance, either as a covert operative or robotic sentry

Inexpensive demonstrations of CENTAUR concepts and capabilities to potential customers (DARPA, NASA, DOE, SWAT, Hazmat Response Teams, Homeland Security, etc.) could begin in the near term by leveraging existing technology and hardware development efforts.

\section{CONCLUSION}

The Centaur concept represents a pairing of two extremely capable robotic systems for the purpose of performing complex manipulation tasks in hazardous surface environments. The concept enables a variety of new mission roles for 
robots assisting human astronauts exploring the surface of the Moon or Mars as well as UGVs accompanying ground troops in the field. Preliminary testing could begin soon by leveraging experience gained with existing, extensively tested prototypes and high TRL technologies. System autonomies, especially in coordinating whole body motions and automating tool use, are important requirements for remote deployment and remain significant challenges.

\section{REFERENCES}

1. R. O. Ambrose, C. Culbert, and F. Rehnmark, “An Experimental Investigation of Dexterous Robots Working with EVA Hardware,” AIAA Space 2001, Albuquerque NM, August 2001.

2. B. Shamah, D. Apostolopoulos, E. Rollins, and W.L. Whittaker, "Field Validation of Nomad's Robotic Locomotion," Proceedings of SPIE - The International Society of Photo-Optical Engineering, Vol. 3525, November 1998.

3. D. Christian et al., "Field Experiments with the Ames Marsokhod Rover," Proceedings of the 1997 Field and Service Robotics Conference, Canberra, Australia, December 1997.

4. J. Jones, "Inflatable Robotics for Planetary Applications," 6th International Symposium on Artificial Intelligence, Robotics, and Automation in Space, I-SAIRAS, Montreal, Canada, June 2001.

5. R.O. Ambrose et al., "Mobile Manipulation using NASA's Segwanaut," International Conference on Robotics \& Automation, New Orleans, Louisiana, April 2004.

6. R.O. Ambrose and R.T. Savely, "Synergy in the Design of Mobile Manipulation," SPIE 2001, Boston, Mass, October 2001.

7. C. Culbert et al., "Activities of the NASA Exploration Team Human-Robotics Working Group," Proceedings of AIAA Space 2003, Long Beach, CA, September 2003. 